\title{
Kernos
}

Revue internationale et pluridisciplinaire de religion grecque antique

$24 \mid 2011$

Varia

\section{Revue des ouvrages reçus à la rédaction}

\section{(2) OpenEdition}

\section{Journals}

Édition électronique

URL : http://journals.openedition.org/kernos/2002

DOI : 10.4000/kernos.2002

ISSN : 2034-7871

\section{Éditeur}

Centre international d'étude de la religion grecque antique

Édition imprimée

Date de publication : 1 janvier 2011

Pagination : 364-367

ISSN : 0776-3824

\section{Référence électronique}

"Revue des ouvrages reçus à la rédaction », Kernos [En ligne], 24 | 2011, mis en ligne le 18 octobre 2011, consulté le 21 septembre 2020. URL : http://journals.openedition.org/kernos/2002 ; DOI : https://doi.org/10.4000/kernos.2002 
"choc des civilisations", p. 573-584; Svetlana Petkova, Les «Trésors cachés»: entre l'intemporalité et l'bistoire, p. 585-598; Anne-Caroline Rendu Loisel, Mémoires et ruines de Mésopotamie, p. 599-614; Thomas Römer, Quand les dieux rendent visite aux hommes (Gn 18-19). Abraham, Lot et la mythologie grecque et proche-orientale, p. 615-626; Youri Volokhine, Pan en Égypte et le «bouc» de Mendès, p. 627-650.

\section{Ouvrages reçus à la rédaction}

ANDÒ Valeria, Cusumano Nicola (éds), Come bestie? Forme e paradossi della violenza tra mondo antico e disagio contemporaneo, Caltanissetta/Roma, Salvatore Sciascia Editore, 2010. 1 vol. $17 \times 24 \mathrm{~cm}, 313$ p. ISBN : 978-88-8241-356-9.

ARYS. Antiguiedad: Religiones y Sociedades, 6 (2003-2005), Universidad de Huelva, 2006. 1 vol. $17 \times$ $23,5 \mathrm{~cm}, 173$ p. ISSN : 1575-166X.

BIERL Anton, Ritual and Performativity: The Chorus in Old Comedy, Cambridge (MA) / London, Harvard UP, 2010² [2009]. 1 vol. $15 \times 23$ cm, XVIII+400 p. (Hellenic Studies, 20). ISBN : 978-0-674-02373-4.

BremMer Jan N., ERSKINE Andrew (éds), The Gods of Ancient Greece. Identities and Transformations, Edinburgh, Edinburgh UP, 2010. 1 vol. $16 \times 24$ cm, XXI+528 p. (Edinburgh Leventis Studies, 5). ISBN : 978-0-7486-3798-0.

BREUiLlot Martine, Continuité et polyvalence : un atout pour l'bellénisme, Strasbourg, Presses universitaires de Strasbourg, 2010. 1 vol. 16,5 × 24 cm, 114 p. (Études orientales, slaves et néo-bellénique). ISBN : 9782-86820-408-0.

BURKERT Walter, Griechische Religion der archaischen und klassischen Epoche, Stuttgart, Kohlhammer, 20112 [1977]. 1 vol. $16 \times 24$ cm, 540 p. (Die Religionen der Menschbeit). ISBN : 978-3-17-021312-8.

Cadmo. Revista de História Antiga. Centro de História da Universidade de Lisboa, 19 (2009), Lisboa, 2009. 1 vol. $16 \times 23 \mathrm{~cm}, 372$ p. ISSN : 0871-9257.

Cadmo. Revista de História Antiga. Centro de História da Universidade de Lisboa, 20 (2010), Lisboa, 2010. 1 vol. $16 \times 23 \mathrm{~cm}, 583$ p. ISSN : 0871-9257.

CALAme Claude, Mythe et Histoire dans l'Antiquité grecque. La création symbolique d'une colonie, Paris, Les Belles Lettres, 2011. 1 vol. $15 \times 21,5$ cm, 288 p. (Vérité des mythes). ISBN : 978-2-251-32459-3.

CARLIER Pierre, Lerouge-COHEN Charlotte, Paysage et religion en Grèce antique. Mélanges offerts à Madeleine Jost, Paris, De Boccard, 2010. 1 vol. $16 \times 23,5$ cm, 272 p. (Travaux de la Maison René-Ginowvès, 10). ISBN : 978-2-7018-0285-5.

CAsadio Giovanni, Johnston Patricia A., Mystic Cults in Magna Grecia, Austin, University of Texas Press, 2009. 1 vol. $16 \times 23,5 \mathrm{~cm}, \mathrm{XV}+372$ p. ISBN : 978-0-292-71902-6.

Charrue Jean-Michel, De l'être et du monde. Ammonius, Plotin, Proclus, Paris, Klincksieck, 2010. 1 vol. 15,5 $\times 24 \mathrm{~cm}, 285$ p. (Études et commentaires, 109). ISBN : 978-2-252-03666-2.

Coppola Daniela, Anemoi: Morphologia dei venti nellimmaginario della Grecia arcaica, Napoli, Liguori Editore, 2010. 1 vol. 15,5 × 21 cm, 147 p. (Università degli Studi di Napoli Federico II. Pubblicazioni del Dipartimento di Discipline Storiche, 24). ISBN : 978-88-207-4346-8.

Dialogues d'bistoire ancienne, 35/2 (2009), Besançon, Presses universitaires de Franche-Comté, 2009. 1 vol. $16 \times 22 \mathrm{~cm}, 249$ p. ISSN : 0755-7256.

Dialogues d'bistoire ancienne, 36/1 (2010), Presses universitaires de Franche-Comté, Besançon, 2010. 1 vol. $16 \times 22 \mathrm{~cm}, 269$ p. ISSN : 0755-7256.

Dialogues d'bistoire ancienne, 36/2 (2010), Presses universitaires de Franche-Comté, Besançon, 2011. 1 vol. $16 \times 22 \mathrm{~cm}, 261$ p. ISSN : 0755-7256.

\footnotetext{
* Nous mentionnons dans cette rubrique tous les ouvrages reçus, en nous réservant la possibilité de ne pas en produire de compte rendu si le sujet n'entre guère dans la thématique de Kernos. Quant aux revues que nous recevons, leurs articles qui concernent la religion grecque, et eux seuls, sont repris dans la Revue des Revues.
} 
Dialogues d'histoire ancienne, supplément 4. Jeux et enjeux de la mise en forme de l'bistoire. Recherches sur le genre historique en Grèce et à Rome, sous la direction de M.-R. Guelfucci, Presses universitaires de FrancheComté, Besançon, 2010. 2 vol. $16 \times 22$ cm, 636 p. ISSN : 0755-7256.

Dialogues d'histoire ancienne, supplément 5. La notion d'empire dans les mondes antiques. Bilan historiographique. Journée de printemps de la SOPHAU - 29 mai 2010, Presses universitaires de Franche-Comté, Besançon, 2011. 1 vol. $16 \times 22 \mathrm{~cm}, 194$ p. ISSN : 0755-7256.

Dimakopoulou Adrienne, Chlôrêis aêdôn pâle rossignol. Une étude sémantique, Paris, Apolis Éditions, 2010. 1 vol. $14,8 \times 21 \mathrm{~cm}, 156$ p. ISBN : 978-2-9532495-3-8.

DOYEN Charles, Poséidon souverain. Contribution à l'bistoire religieuse de la Grèce mycénienne et archaïque, Bruxelles, Académie Royale de Belgique, 2011. 1 vol. $16 \times 24 \mathrm{~cm}, 391$ p. (Mémoire de la Classe des Lettres in- $8^{\circ}, 3^{\mathrm{e}}$ série, tome $\left.55, \mathrm{n}^{\circ} 2072\right)$. ISBN : 978-2-8031-0279-2.

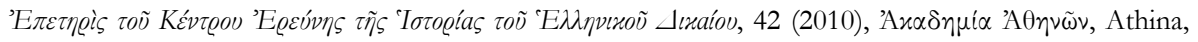
2010. 1 vol. $17,5 \times 24 \mathrm{~cm}, 268$ p. ISSN : 1105-0055.

Friesen Steven J., SCHOwalter Daniel N., Walters James C. (éds), Corinth in Context. Comparative Studies on Religion and Society, Leiden/Boston, Brill, 2010. 1 vol. 16,5 × 24,5 cm, 517 p. (Supplements to Novum Testamentum, 134). ISBN : 978-90-04-18197-7.

Gaia. Revue interdisciplinaire sur la Grèce archä̈que 13 (2010), Grenoble, ERGA (Université StendhalGrenoble 3), 2010.1 vol. $16 \times 24$ cm, 244 p. ISSN : 1269-3717.

Harissis Haralampos V., HARISSIS Anastasios V., Apiculture in the Prehistoric Aegean. Minoan and Mycenaean Symbols Revisited, Oxford, British Archaeological Reports, 2009. 1 vol. $21 \times 29,7$ cm, 95 p. (BAR International Series, 1958). ISBN : 978-1-4073-0454-0.

Haysom Matthew \& Wallensten Jenny (ed.), Current Approaches to Religion in Ancient Greece, Papers presented at a symposium at the Swedish Institute at Athens, 17-19 April 2008, Stockholm, 2011. 1 vol. $16,5 \times 24$ cm, 315 p. (Acta Instituti Atheniensis Regni Sueciae, Series in 8, 21). ISBN : 978-917916-059-3.

Incidenza dell'antico. Dioaloghi di storia greca, 8 (2010), Luciano Editore, Napoli, 2010. 1 vol. $16 \times 23,5 \mathrm{~cm}$, 262 p. ISSN : 1971-2995.

Ismard Paulin, La cité des réseaux. Athènes et ses associations, VIe-Ier siècle av. J.-C., Paris, Publications de la Sorbonne, 2010. 1 vol. $16 \times 24$ cm, 522 p. ISBN : 978-2-85944-653-6.

Kaplanian Patrick, Mythes grecs d'origine. I - Prométhée et Pandore, Paris, éditions de l'Adret, 2011.1 vol. 13,5 × 21,5 cm, 448 p. (L'Entreligne, Ethnologie). ISBN : 978-2-909623-06-1.

KNOEPFler Denis, La Patrie de Narcisse. Un héros mythique enraciné dans le sol et dans l'bistoire d'une cité grecque, Paris, Odile Jacob, 2010. 1 vol. 14,5 × 22 cm, 238 p. (Collège de France). ISBN : 978-2-73812500-2.

LindBlom Michael, Wells Berit (éds), Mastos in the Berbati Valley. An Intensive Archaeological Survey, Stockholm, 2011. 1 vol. $21,5 \times 27,5 \mathrm{~cm}, 196$ p. (Skrifter utgivna av Svenska Institutet $i$ Athen, $\left.4^{\circ}, 54\right)$. ISBN : 978-91-7916-058-6.

LuCCI Carlamaria, Le diverse percezioni del tempo nell'epica greca arcaica: Studi sull'Iliade e l'Odissea, Pisa, Edizioni ETS, 2011. 1 vol. 15,5 × 22,5 cm, 287 p. (ANTHROPOI. Studi e materiali di Antropologia storica del mondo antico, 7). ISBN : 978-884672955-2.

MARRUCCI Lucia, Kratos e arche: Funzioni drammatiche del potere, Amsterdam, Adolf M. Hakkert Editore, 2010. 1 vol. $17,6 \times 25$ cm, 308 p. ISBN : 978-90-256-1255-9.

Montepaone Claudia (éd.), Pitagoriche. Scruta femminili di età ellenistica, Bari, Edipuglia, 2011. 1 vol. $17 \times$ $24 \mathrm{~cm}, 128$ p. (Collana del Dipartimento di Scienze dell'antichità dell'Università di Bari, sezione storica, 46). ISBN : 978-88-7228-620-3.

MORARD Thomas, Horizontalité et verticalité. Le bandeau bumain et le bandeau divin chez le Peintre de Darius, Mainz, von Zabern, 2009. 1 vol. 21,5 × 30 cm, X+264 p., 120 pl. ISBN : 978-8053-3965-0.

MylonOPOUlos Joannis (éd.), Divine Images and Human Imaginations in Ancient Greece and Rome, Leiden/Boston, Brill, 2010. 1 vol. 16,5 x 24,5 cm., ill., 437 p., (Religions in the Graeco-Roman World, 170). ISBN : 978-90-04-17930-1.

Mythos. Rivista di Storia delle Religioni, n.s. 4 (2010), Palermo, Università degli Studi di Palermo, Dipartimento di Beni Culturali, Sezione di Storia Antica, Salvatore Sciascia Editore, 2011. 1 vol. 17 $\times 24 \mathrm{~cm}, 229$ p. ISSN : 1972-2516. 
NERI Francesco, Reliquie eroiche nella Grecia arcaica e classica (VI - V sec. A.C.), Napoli, Istituto Italiano per gli Studi Storici, 2010. 1 vol. 16,5 × 24 cm, 255 p. ISBN : 978-88-15-13979-5.

Opuscula. Annual of the Swedish Institutes at Athens and Rome, 2 (2009), Stockholm, Svenska Institutet i Athen och Svenska Institutet i Rom, 2009. 1 vol. $21 \times 27$ cm, 232 p. ISSN : 2000-0898.

Opuscula. Annual of the Swedish Institutes at Athens and Rome, 3 (2010), Stockholm, Svenska Institutet i Athen och Svenska Institutet i Rom, 2010.1 vol. $21 \times 27$ cm, 224 p. ISSN : 2000-0898.

Pallas. Revue d'études antiques, 82 (2010). Ab Aquitania in Hispaniam. Mélanges d'bistoire et d'archéologie offerts à Pierre Sillières, Toulouse, Presses universitaires du Mirail, 2010. 1 vol. $16 \times 24 \mathrm{~cm}, 521$ p. ISSN : 0031-0387/ISBN : 978-2-8107-0101-8.

Pallas. Revue d'études antiques, 83 (2010). Glyptique romaine, Toulouse, Presses universitaires du Mirail, 2010. 1 vol. $16 \times 24$ cm, 339 p. ISSN : 0031-0387/ISBN : 978-2-8107-0103-2.

Pallas. Revue d'études antiques, 84 (2010). Viure en Gaule Narbonnaise. Isis au Capitole, Toulouse, Presses universitaires du Mirail, 2010. 1 vol. $16 \times 24$ cm, 467 p. ISSN : 0031-0387/ISBN : 978-2-8107-0102-5.

PARKER Robert, On Greek Religion, Ithaca/London, Cornell UP, 2011.1 vol. 15,5 × 23,5 cm, 328 p. (Cornell Studies in Classical Philology). ISBN : 978-0-8014-4948-2 (cloth printing) ou 978-0-8014-77355 (paperback printing).

PEDrucci Giulia, Cibele Frigia e la Sicilia. I santuari rupestri nel culto delle dea, Roma, «L'Erma» di Bretschneider, 2009. 1 vol. 17,5 × 24,5 cm, 156 p., 21 pl. (Studia Archeologica, 168). ISBN : 978-888265-560-0.

PETIT Thierry, Edipe et le Chérubin. Les sphinx levantins, cypriotes et grecs comme gardiens d'immortalité, Fribourg/Göttingen, Academic Press Fribourg et Vandenhoeck \& Ruprecht, 2011. 1 vol. $16 \times$ 23,5 cm, 388 p. (Orbis Biblicus et Orientalis, 248). ISBN : 978-3-7278-1692-5 (Academic Press Fribourg) ou 978-3-525-54369-6 (Vandenhoeck \& Ruprecht).

Philotheos. International Journal for Philosophy and Theology, 10 (2010), Beograd, 2010. 1 vol. $17 \times 24 \mathrm{~cm}$, 367 p. ISSN : 1451-3455.

PRESCENDI Francesca, VOLOKHINE Youri (éds), Dans le laboratoire de l'historien des religions. Mélanges offerts à Philippe Borgeaud, Genève, Labor et Fides, 2011. 1 vol. $15 \times 22,5 \mathrm{~cm}, 668$ p. (Religions en perspective, 24). ISBN : 978-2-8309-1428-3.

Pseudo-Dionigi di Alicarnasso, I discorsi figurati I e II (Ars Reth. VIII e IX Us.-Rad.), Introduzione, traduzione e commento a cura di Stefano Dentice di Accadia, Pisa/Roma, Fabrizio Serra Editore, 2010. 1 vol. $17 \times 24$ cm, 184 p. (coll. Aion. Quaderni 14). ISBN : 978-88-6227-304-6.

Robertson Noël, Religion and Reconciliation in Greek Cities. The Sacred Laws of Selinus and Cyrene, Oxford, Oxford UP, 2010. 1 vol. $16 \times 24$ cm, 414 p. (APA American Classical Studies, 54). ISBN : 978-0-19539400-9.

RÜPKE Ulrike, RÜPKE Jörg, Die 101 wichtigsten Fragen. Götter und Mythen der Antike, München, C.H. Beck, 2010. 1 vol. $12,5 \times 19$ cm, 160 p. ISBN : 978-3-406-60630-4.

SCARPI Paolo, Si fa presto a dire Dio. Riflessioni sul multiculturalismo religioso, Milano, Adriano Salani Editore S.p.A., 2010. 1 vol. 13,5 × 20,5 cm, 149 p. ISBN : 978-88-6220-120-9.

SCHudDeBOOM Feyo L., Greek. Religious Terminology - Telete \& Orgia. A Revised and Expanded English Edition of the Studies by Zijderveld and Van der Burg, Leiden/Boston, Brill, 2009. 1 vol. 16,5 $\times$ 24,5 cm, 285 p. (Religions in the Graeco-Roman World, 169). ISBN 978-90-04-178137.

SCROFAnI Giorgio, La religione impura. La riforma di Giuliano Imperatore, Paideia Editrice, Brescia, 2010. 1 vol. 13,5 × $21 \mathrm{~cm}, 190$ p. (Studi Biblici, 163). ISBN : 978-88-394-0777-1.

SFAMENi GASPARro Giulia, Dio unico, pluralità e monarchia divina. Esperienze religiose e teologie nel mondo tardo-antico, Brescia, Morcelliana, 2010. 1 vol. 15,5 × 22,5 cm, 280 p. (Scienze e storia delle religioni). ISBN : 978-88-372-2433-2.

Smith Amy C., Pickup Sadie (éds), Brill's Companion to Aphrodite, Leiden/Boston, Brill, 2010.1 vol. $16,5 \times 24,5 \mathrm{~cm}, 452$ p. ISBN : 978-9004-18003-1.

Sourvinou-Inwood Christiane, Athenian Myths \& Festivals. Aglauros, Erechtheus, Plynteria, Panathenaia, Dionysia, Oxford, Oxford UP, 2011. 1 vol. 14,5 × 22 cm, 377 p. ISBN : 978-0-19-959207-4.

Sundahl Mark, Mirhady David, Arnaoutoglou Ilias, A New Working Bibliography of Ancient Greek Law (7th-4 th centuries BC), Athens, Academy of Athens, 2011. 1 vol. 17,5 $\times 24 \mathrm{~cm}, 657$ p. (Yearbook of the Research Centre for the History of Greek Law, volume 42, suppl. XI). ISBN : 978-960-404-198-5. 
TOSETTI Giovanni, Unioni divino-umane. Un percorso storico-religioso nel mito greco arcaico, Cosenza, Edizioni Lionello Giordano, 2008. 1 vol. $14,5 \times 20,5 \mathrm{~cm}$, VII+515 p. (HIER A. Collana di studi storico-religiosi, 10). ISBN : 978-88-86919-26-3.

VEYMIERS Richard, "Hileôs tôi phorounti”. Sérapis sur les gemmes et les bijoux antiques, Bruxelles, Académie royale de Belgique, 2009. 1 vol. 22,5 × 28,5 cm, 608 p. (Classe des Lettres). ISBN : 978-28031-0261-7.

Villanueva Puig Marie-Christine, Corpus Vasorum Antiquorum. Musée du Louvre, fascicule 28 : Les lécythes attiques à figures noires, Paris, De Boccard, 2010. 1 vol. $24,5 \times 32 \mathrm{~cm}, 61$ p., 55 pl. (Académie des Inscriptions et Belles-Lettres). ISBN : 978-28775424-94. 